Article

\title{
Lactobacillus plantarum with Broad Antifungal Activity as a Protective Starter Culture for Bread Production
}

\author{
Pasquale Russo $^{1,2, *}$, Clara Fares ${ }^{3}{ }^{(\mathbb{D}}$, Angela Longo ${ }^{1}$, Giuseppe Spano ${ }^{1}$ and Vittorio Capozzi ${ }^{1}$ \\ 1 Department of Science of Agriculture, Food and Environment, University of Foggia, Via Napoli 25 , \\ 71122 Foggia, Italy; angela.longo@unifg.it (A.L.); giuseppe.spano@unifg.it (G.S.); \\ vittorio.capozzi@unifg.it (V.C.) \\ 2 Promis Biotech Via Napoli 25, 71122 Foggia, Italy \\ 3 Council for Agricultural Research and Economics-Research Centre for Cereal and Industrial Crops \\ (CREA-CI), S.S.673 km 25.200, 71122 Foggia, Italy; clara.fares@crea.gov.it \\ * Correspondence: pasquale.russo@unifg.it; Tel.: +39-0881-589-303
}

Received: 13 November 2017; Accepted: 4 December 2017; Published: 11 December 2017

\begin{abstract}
Bread is a staple food consumed worldwide on a daily basis. Fungal contamination of bread is a critical concern for producers since it is related to important economic losses and safety hazards due to the negative impact of sensorial quality and to the potential occurrence of mycotoxins. In this work, Lactobacillus plantarum UFG 121, a strain with characterized broad antifungal activity, was analyzed as a potential protective culture for bread production. Six different molds belonging to Aspergillus spp., Penicillium spp., and Fusarium culmorum were used to artificially contaminate bread produced with two experimental modes: (i) inoculation of the dough with a commercial Saccharomyces cerevisiae strain (control) and (ii) co-inoculation of the dough with the commercial S. cerevisiae strain and with L. plantarum UFG 121 . L. plantarum strain completely inhibited the growth of F. culmorum after one week of storage. The lactic acid bacterium modulated the mold growth in samples contaminated with Aspergillus flavus, Penicillium chrysogenum, and Penicillium expansum, while no antagonistic effect was found against Aspergillus niger and Penicillium roqueforti. These results indicate the potential of L. plantarum UFG 121 as a biocontrol agent in bread production and suggest a species- or strain-depending sensitivity of the molds to the same microbial-based control strategy.
\end{abstract}

Keywords: antifungal; bioprotection; bread; Lactobacillus plantarum; phenyllactic acid; Aspergillus; Penicillium; Fusarium

\section{Introduction}

Bread, obtained by baking a fermented dough of cereals flour, water, and other ingredients, is ancient and, due to its nutritional properties and low price, is a staple of many diets and an essential contributor of energy and nutritional intake in both developed and developing countries [1,2]. Microbial alteration of bread is a critical concern for bakeries, and it is mainly attributable to the development of spoilage molds. Apart from significant economic losses due to the negative impact on sensory properties, the occurrence of filamentous fungi poses a safety hazard for human health due to the potential ability of some fungal strains to produce mycotoxins [3,4]. Moreover, fungal spoilage control is critical for the extension of the shelf life of bakery goods, especially from an industrial perspective [5]. Traditionally, the shelf life of bread has been extended by the addition of chemical preservatives such as ethanol and weak organic acids, mainly propionic, sorbic, benzoic, and acetic acid and their salts [6]. As an alternative, physical methods such as microwave and infrared radiation and innovative packaging technologies have been exploited to reduce fungal developments in bakery 
products [7]. However, a strong societal demand, supported by public authorities, has urged more eco-friendly approaches mainly relying on the use of essential oils and antagonistic microorganisms as preservation tools [8]. In this context, lactic acid bacteria (LAB) have the greatest appeal as biocontrol agents due to their status of food-grade microorganisms $[9,10]$. The antifungal ability of some LAB strains is owed to the production of secondary metabolites, mainly including lactic acid and other organic acids, phenolic compounds, carbon dioxide, ethanol, hydrogen peroxide, fatty acids, acetoin, diacetyl, and cyclic dipeptides [11-13]. Moreover, synergistic interactions among different bioactive molecules could substantially increase the overall LAB antimicrobial activity [14]. Sourdough is a valuable and comprehensive source of antifungal and mycotoxin-controlling compounds synthesized by LAB during fermentation [15-17]. In the last few years, several LAB strains belonging to the species Lactobacillus amylovorus, Lactobacillus reuteri, Lactobacillus brevis, Lactobacillus plantarum, Lactobacillus rossiae, and Lactobacillus paralimentarius have been proposed as starter protective cultures to enhance the shelf life of bread [18-22]. Antifungal properties against bakery product spoilage molds have also been shown by Propionibacterium cultures [23]. Moreover, the ability of antagonistic yeasts to control fungal contamination in bread has been investigated in Meyerozyma guilliermondii and Wickerhamomyces anomalus [24,25]. In particular, Penicillium roqueforti was delayed until 14 days of storage in bread produced with a combination of these antifungal yeasts and of a specific L. plantarum strain [25]. Furthermore, proteinaceous compounds from different food matrices or legumes flour hydrolysates could be used as ingredients in the bakery industry to enhance the antifungal properties of sourdoughs [26,27].

In this work, a strain of L. plantarum previously characterized for its antifungal potential [28] was investigated for its ability to control the growth of six different species of filamentous fungi belonging to three different genera in artificially contaminated bread after one week of storage.

\section{Materials and Methods}

\subsection{Microbial Strains and Growth Conditions}

Lactobacillus plantarum UFG 121 was available at the Laboratory of Industrial Microbiology of the University of Foggia (Foggia, Italy) and routinely grown in de Man Rogosa Sharpe (MRS) broth (Oxoid, Basingstoke, UK) at $30^{\circ} \mathrm{C}$ for $24 \mathrm{~h}$.

Six filamentous fungi, namely Penicillium roqueforti CECT 20508, Penicillium expansum CECT 2278, Penicillium chrysogenum CECT 2669, Aspergillus niger CECT 2805, Aspergillus flavus CECT 20802, and Fusarium culmorum CECT 2148 were provided by the Spanish Type Culture Collection (CECT, Paterna, Spain). Fungal cultures were plated on malt extract agar (Oxoid) and incubated at $24{ }^{\circ} \mathrm{C}$ for 5 days.

The commercial fresh yeast Saccharomyces cerevisiae "Lievital" (Lessafre, Marcq-en-Baroeul, France) was resuspended in sterile saline solution, streaked on plates of yeast extract, peptone, dextrose (YPD, Oxoid), and incubated at $30{ }^{\circ} \mathrm{C}$ for $48 \mathrm{~h}$. Then, a single colony was resuspended in YPD broth and incubated at $30^{\circ} \mathrm{C}$.

\subsection{Dough and Sourdough Preparation}

Cells at exponential phase of S. cerevisiae and L. plantarum UFG 121 were recovered by centrifugation $(5000 \times g, 5 \mathrm{~min})$, washed twice with sterile saline solution, and resuspended in sterile water. For sourdough preparation, microbial starters were inoculated at a concentration of about $10^{8}$ and $10^{6} \mathrm{cfu}^{-1}$ for L. plantarum and yeast respectively, in a mixture of wheat flour type " 0 " Manitoba (initial moisture of $11.9 \% w / w$, supplied by Lo Conte, IPAFOOD, Ariano Irpino, Italy) and water $(37.5 \% w / w)$ containing sucrose $(6 \%), \mathrm{NaCl}(3 \% w / w)$, and animal fats $(3 \% w / w)$. A dough control sample was inoculated only with the commercial yeast at a final concentration of about $10^{6} \mathrm{cfu} \mathrm{g}^{-1}$. The fermentation was carried out at $30^{\circ} \mathrm{C}$ for $18 \mathrm{~h}$. 


\subsection{Bread Production}

Bread was obtained in a pilot plant according to the methods ACC10-10B29 modified by Capozzi et al. [29]. Briefly, the sourdough was subject to kneading for $10 \mathrm{~min}$, followed by fermentation for $90 \mathrm{~min}$ at room temperature. The sourdough was then aliquoted in samples of about $250 \mathrm{~g}$ each and fermented for another $90 \mathrm{~min}$. Afterwards, samples were put into apposite shapes to attain specific dimensions $(11 \mathrm{~cm} \times 25 \mathrm{~cm} \times 7 \mathrm{~cm})$ and submitted to a final fermentation step of $90 \mathrm{~min}$ at room temperature. Bread was obtained after the dough was baked in an oven at $220{ }^{\circ} \mathrm{C}$ for $40 \mathrm{~min}$. The bread was cut in half in order to repeat each experimental condition in duplicate.

\subsection{Artificial Contamination of Bread}

A preparation of fungal spores was obtained by brushing with a sterile swab the plate surface of each five-day-grown mold. Spores were resuspended in sterile distilled water and concentrated at $8 \times 10^{4}$ spores $\mathrm{mL}^{-1}$. Artificially contaminated samples were obtained by spraying $15 \mathrm{~mL}$ of the spore solution on the surface of the bread. Samples were immediately packaged using polyethylene terephthalate bags and stored for 7 days at room temperature. At this time, the in vivo antagonistic activity against each tested mold was qualitatively determined by comparing the area contaminated by the spoilage fungi in bread fermented with the starter yeast or co-fermented with L. plantarum UFG 121. Results were expressed according to the following scale: no/low $(-)$, moderate $(+)$, high $(++)$, and very high $(+++)$ inhibition activity, if the area covered by each filamentous fungi in bread co-fermented with UFG 121 strain was reduced in the ranges $0-25 \%, 25-50 \%, 50-75 \%$, and $75-100 \%$, respectively.

\subsection{Sensorial Quality}

The sensorial analysis was carried out at our laboratory by a panel of six panelists before the artificial contamination of the samples with the spoilage molds. Panelists were previously trained in order to recognize and score the analyzed quality descriptors. Samples were coded with a random 3-digit number in order to minimize subjectivity. The sensorial attributes evaluated were overall appearance, aromatic profile, off odor, color, softness, and texture. Every attribute was scored on a $1-5$ hedonic scale, where 1 = atypical, undesirable, and $5=$ typical, desired. Sensorial trials were performed on six samples for each treatment.

\subsection{Statistical Analysis}

The quality descriptors analyzed in the sensorial trials were subjected to one-way analysis of variance (ANOVA). Pairwise comparison of treatment means was achieved by Tukey's procedure, with a significance level of $p \leq 0.05$, using the statistical software Past 3.0 (University of Oslo, Oslo, Norway).

\section{Results}

\subsection{Production of Bread Co-Fermented with a Protective L. plantarum Strain}

Bread samples were produced by fermentation of the dough with a commercial yeast commonly used in breadmaking, or by its co-inoculation with L. plantarum UFG 121, a strain with a characterized antifungal activity [28]. A preliminary qualitative analysis was performed during the breadmaking process in order to detect if the employment of the protective strain L. plantarum UFG 121 as a starter culture could affect the bread production from a technological and/or sensorial point of view. As reported in Figure 1, some differences were observed among samples inoculated with the commercial yeast starter or co-inoculated with UFG 121 strain. In particular, after $18 \mathrm{~h}$ of the fermentation step, the dough was apparently softer when fermented with $S$. cerevisiae compared with the co-inoculation approach using the protective L. plantarum strain (Figure 1A). In contrast, the sourdough developed a more complex aromatic profile when co-fermented with UFG 121 strain. In agreement with this finding, after kneading the dough fermented using the yeast starter, the dough 
was more compact and elastic and therefore easier to break and to produce the desired shape. Co-fermentation with yeast and L. plantarum resulted in a sourdough with an inhomogeneous texture, which could be disadvantageous for subsequent processing (Figure 1B). However, no important differences were detected after the final fermentation, since both samples were well compacted and leavened with a smooth surface (Figure 1C). After all samples were baked, similar features in terms of color, softness, and texture were apparent. However, samples co-inoculated with L. plantarum UFG 121 presented more pronounced alveolation (Figures 1D and 2). Moreover, sensorial analysis indicated the absence of off odors in both breads, while the aromatic profile of the bread inoculated with L. plantarum UFG 121 scored higher than the control bread. In general, the overall appearance of the bread obtained via UFG 121 co-fermentation was perceived as slightly better by the panelists.
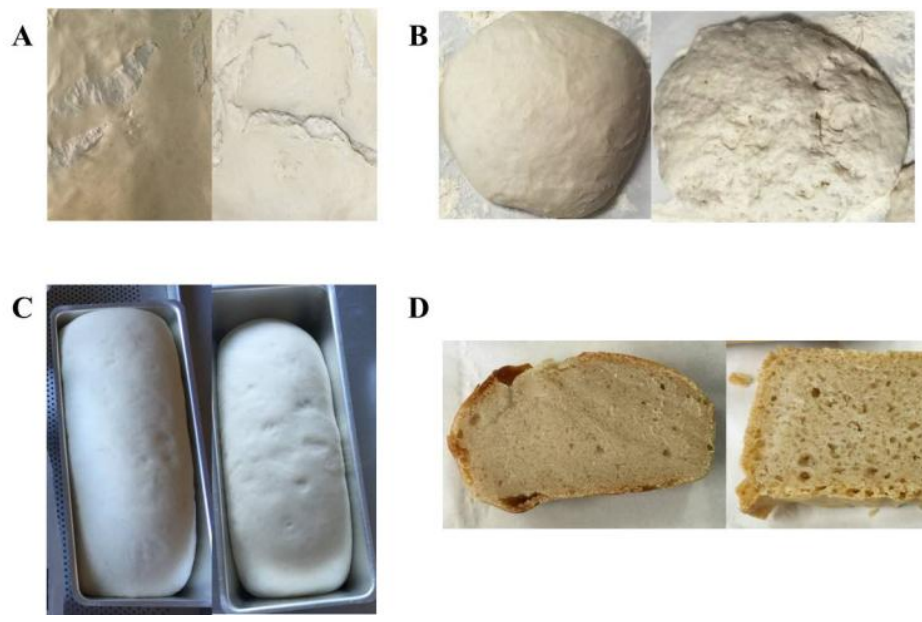

D

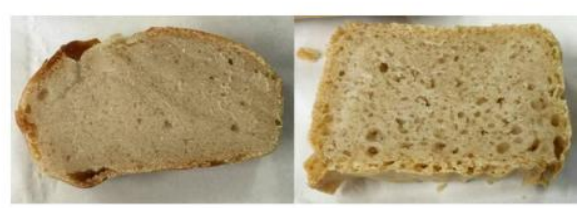

Figure 1. Dough fermented by S. cerevisiae "Lievital" (left pictures) or co-fermented with L. plantarum UFG 121 (right pictures) after (A) first fermentation step for $18 \mathrm{~h}$ at $30^{\circ} \mathrm{C}$; (B) kneading and shaping; (C) the final fermentation step for $90 \mathrm{~min}$ at room temperature; and (D) baking and cutting.

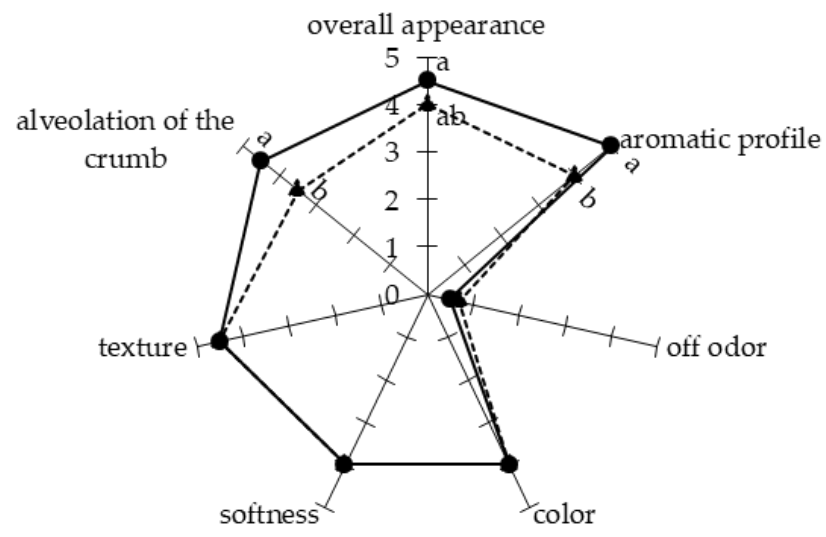

Figure 2. Sensory attributes of bread obtained by fermentation with S. cerevisiae "Lievital" (triangle, dashed line) or co-fermented with L. plantarum UFG 121 (circle, continuous line). Reported values are means of six independent replicates, and they are expressed by using a hedonic scale from 1 to 5 ( 1 = atypical, undesirable; 5 = typical, desired). Means with different letters are significantly different according to Tukey's test $(p \leq 0.05)$.

\subsection{Analysis of the Protective Potential of L. plantarum UFG 121 in Artificially Contaminated Bread}

With the aim to evaluate the potential of L. plantarum UFG 121 as a culture protective against typical molds on bakery products, bread samples were artificially contaminated by spraying a concentrated spore suspension onto the bread slices. After one week of storage, the in vivo antagonistic 
activity against each tested mold was qualitatively determined by comparing the area covered by the spoilage fungi in the two tested experimental modes: (1) bread fermented with the commercial $S$. cerevisiae and (2) co-fermented with L. plantarum UFG 121 (Figure 3). As shown in Figure 3, after one week of storage the surface of the control bread artificially contaminated were always wholly covered by the molds. In contrast, different scenarios were observed when bread samples were co-fermented with L. plantarum UFG 121. In particular, no inhibition was found in bread samples artificially inoculated with $A$. niger and $P$. roquefort $i$ that appeared completely contaminated by both the molds (Figure 3A,B). A moderate in vivo antagonistic activity was detected against $P$. chrysogenum and $P$. expansum whose growth was limited in samples obtained with UFG 121 strain (Figure 3C,D). In contrast, a higher protective effect was observed in samples contaminated by A. flavus in which only approximately $20 \%$ of the bread surface was covered by the spoilage (Figure 3E). Interestingly, no development of F. culmorum was observed, suggesting that the employment of L. plantarum UFG 121 during bread fermentation was a successful strategy to thoroughly inhibit F. culmorum growth (Figure 3F).

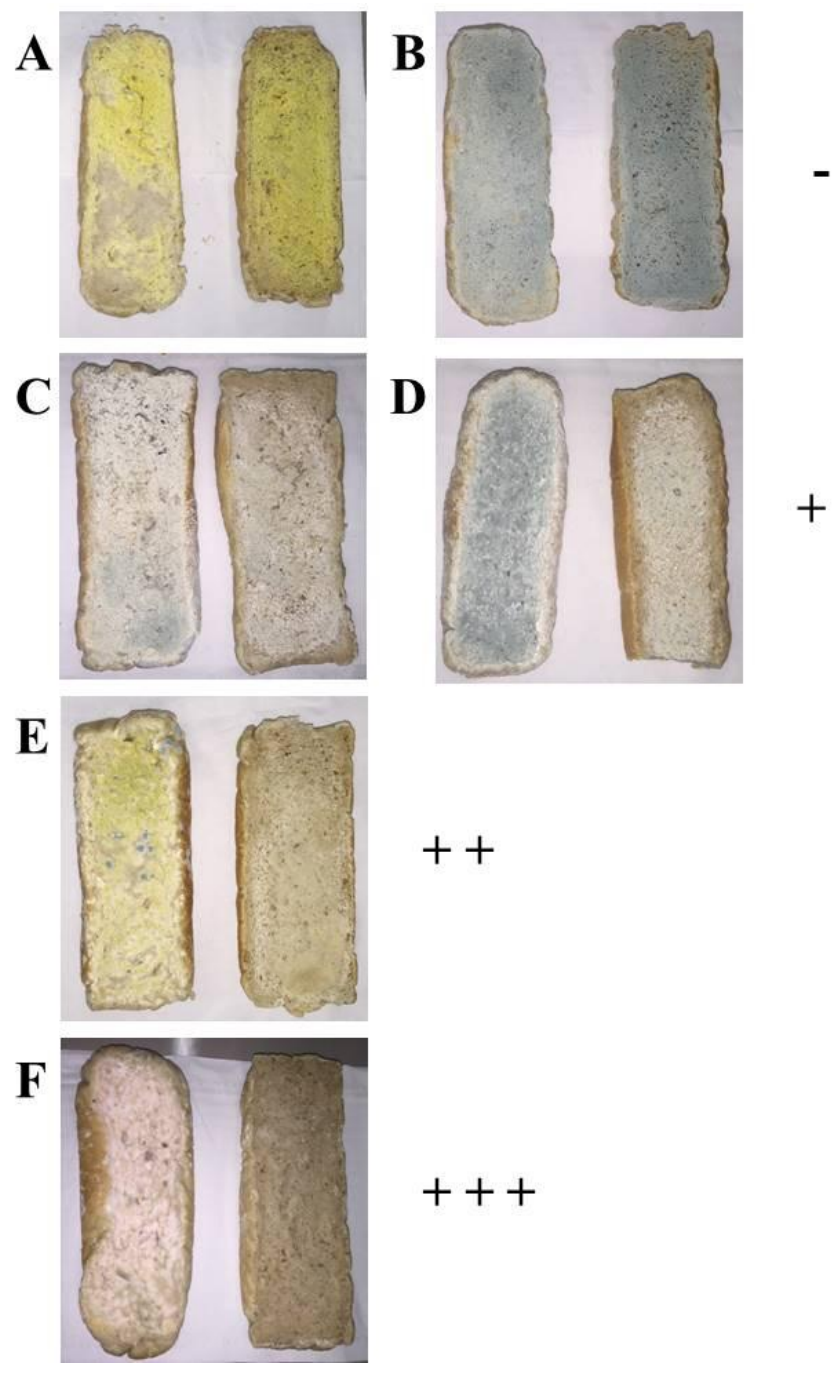

Figure 3. Bread obtained by fermentation with S. cerevisiae "Lievital" (left pictures) or co-fermented with L. plantarum UFG 121 (right pictures) after one week of storage at room temperature and artificially contaminated with A. niger CECT 2805 (A); P. roqueforti CECT 20508 (B); P. chrysogenum CECT 2669 (C); P. expansum CECT 2278 (D); A. flavus CECT 20802 (E); F. culmorum CECT 2148 (F). Antifungal activity was expressed as no/low $(-)$, moderate $(+)$, high $(++)$, and very high $(+++)$, when the contaminated area was reduced in the ranges $0-25 \%, 25-50 \%, 50-75 \%$, and $75-100 \%$, respectively. 


\section{Discussion}

In the present study, six different molds belonging to Aspergillus spp., Penicillium spp., and Fusarium culmorum were used to artificially contaminate bread produced by fermentation of the dough with a commercial S. cerevisiae, or by its co-inoculation with L. plantarum UFG 121 . The fungal strains were selected because they are representative molds of bread spoilage [30] and they have an ability to produce mycotoxins. In particular, A. flavus CECT 20802 produced aflatoxins B1, B2, M1, M2; F. culmorum CECT 2148 fusarine C; P. roqueforti CECT 20508 was able to synthesize PR toxin; while P. expansum CECT 2278 was responsible for the production of patulin and citrinin. In the present study, L. plantarum UFG 121, previously characterized for its broad antifungal activity against these mold strains [28], was investigated as a protective culture for bread production.

The employment of LAB strains with antagonistic activity has been widely proposed as an innovative green strategy to fight spoilage filamentous fungi in order to enhance the shelf life of bakery products [24,31-33]. In general, the antifungal effect has been attributed to the production of some secondary metabolites during the fermentation of sourdough [11]. Therefore, according to previous studies [24,34], in this work, a fermentation step of $18 \mathrm{~h}$ was performed in order to allow the UFG 121 strain to enrich the sourdoughs with bioactive antifungal compounds. However, differences between the control samples and the bread obtained by co-fermentation with L. plantarum UFG 121 were qualitatively detected as the bread was made that might have affected the production process. Nonetheless, as previous reported by Coda et al. [25], co-fermentation of the dough with yeast and selected LAB resulted in bread showing good chemical and textural features, including elasticity, color, and alveolation. Moreover, an improvement in terms of complexity of the aromatic profile was noted in the bread co-fermented with UFG 121. In agreement with this finding, Makhoul et al. [35], analyzing pro-technological microbe/matrix interactions during food fermentation, reported a greater impact of the microbial fraction on the volatile organic compounds of bread. However, the present work is only a preliminary study that should be further complemented by the analytical determination of the main physico-chemical parameters as well as the impact of a protective LAB culture on the organoleptic profile of the bread [36,37].

In our previous study, we found that the preservative potential of L. plantarum UFG 121 was mainly due to the production of lactic acid and phenyllactic acid [28]. Phenyllactic acid production by L. plantarum has been linked to the antifungal activity against fungal strains isolated from bakery products belonging to species of Aspergillus, Penicillium, and Fusarium [38]. Similarly, an increase in the shelf life of bread obtained by fermentation with L. plantarum CRL 778 and artificially contaminated with Penicillium spp. has been found to be related to the synthesis of acetic and phenyllactic acid as well as lactic acid [20]. Moreover, organic acids including phenyllactic acid from a strain of L. amylovorus have been found to be responsible for a higher shelf life in gluten-free breads [21]. Lactic acid, phenyllactic acid, and two cyclic dipeptides found in sourdoughs fermented by L. plantarum FST 1.7 have been identified as the main antifungal compounds able to retard the growth of F. culmorum and Fusarium graminearum on bread [18].

In a similar way, in the present study, F. culmorum CECT 2148 was completely inhibited in bread fermented by L. plantarum UFG 121. The in vitro assays performed suggest that CECT 2148 was the most sensitive mold when exposed to UFG 121 cell-free supernatant [28]. Moreover, this result has been confirmed in situ, since fermentation by UFG 121 and artificial contamination with CECT 2148 (after thermal stabilization) increased the shelf life of an oat-based formulation from less than one week to the second week of cold storage, indicating that a strong bioprotection could be provide by antifungal compounds produced in a $16 \mathrm{~h}$ fermentation step [28]. In contrast, fermentation with L. plantarum UFG 121 had no effect in countering the growth of P. roqueforti CECT 20508 and A. niger CECT 2805, but reduced bread contamination by the remaining tested molds to different extents. Interestingly, these results were only partially predicted by the in vitro assays [28], suggesting that interactions with the commercial yeast, process parameters, and/or the food matrix might modulate the antagonistic activity of selected LAB against fungal strains. Therefore, further investigations are 
required to establish the effectiveness of the antifungal compounds, their synergistic interactions, and the complex microbial and physico-chemical relationships occurring in the food environment.

\section{Conclusions}

In recent years, several studies have aimed to enhance the shelf life and safety of bakery products by analyzing the antifungal potential of protective microbial starter cultures and the corresponding sourdough. However, most of these works have been performed using only one or a few mold strains as fungal indicators. In this study, although with a preliminary experimental plan, we analyzed the in situ bioprotection effectiveness of a L. plantarum strain against a representative panel of six different mold species belonging to three different genera generally recognized as being mainly responsible for the natural contamination of bakery foods. Our results indicated in these molds a species- or strain-dependent sensitivity to the same microbial-based control strategy, suggesting that a new generation of mixed starter protective cultures, active against different fungal species, might be better able to globally extend the shelf life of baked goods.

Acknowledgments: Vittorio Capozzi was supported by Fondo di Sviluppo e Coesione 2007-2013-APQ Ricerca Regione Puglia "Programma regionale a sostegno della specializzazione intelligente e della sostenibilità sociale ed ambientale-FutureInResearch". Pasquale Russo was supported by a grant of the Apulian Region in the framework of "Perform Tech (Puglia Emerging Food Technology)" project (practice code LPIJ9P2).

Author Contributions: P.R., V.C., and G.S. conceived and designed the experiments; A.L. performed the experiments; P.R. and V.C. analyzed the data; C.F. contributed to the bread production in the pilot plant; P.R. wrote the paper; G.S. and V.C. critically revised the manuscript.

Conflicts of Interest: The authors declare no conflict of interest.

\section{References}

1. Guyot, J.P. Cereal-based fermented foods in developing countries: Ancient foods for modern research. Int. J. Food Sci. Technol. 2012, 47, 1109-1114. [CrossRef]

2. Silow, C.; Axel, C.; Zannini, E.; Arendt, E.K. Current status of salt reduction in bread and bakery products-A review. J. Cereal Sci. 2016, 72, 135-145. [CrossRef]

3. Vaclavikova, M.; Malachova, A.; Veprikova, Z.; Dzuman, Z.; Zachariasova, M.; Hajslova, J. 'Emerging' mycotoxins in cereals processing chains: Changes of enniatins during beer and bread making. Food Chem. 2013, 136, 750-757. [CrossRef] [PubMed]

4. Lee, H.J.; Ryu, D. Worldwide occurrence of mycotoxins in cereals and cereal-derived food products: Public health perspectives of their co-occurrence. J. Agric. Food Chem. 2017, 65, 7034-7051. [CrossRef] [PubMed]

5. Almeida, E.L.; Steel, C.J.; Chang, Y.K. Par-baked bread technology: Formulation and process studies to improve quality. Crit. Rev. Food Sci. Nutr. 2016, 56, 70-81. [CrossRef] [PubMed]

6. Samapundo, S.; Devlieghere, F.; Vroman, A.; Eeckhout, M. Antifungal properties of fermentates and their potential to replace sorbate and propionate in pound cake. Int. J. Food Microbiol. 2016, 237, 157-163. [CrossRef] [PubMed]

7. Van Long, N.N.; Joly, C.; Dantigny, P. Active packaging with antifungal activities. Int. J. Food Microbiol. 2016, 220, 73-90. [CrossRef] [PubMed]

8. Salas, M.L.; Mounier, J.; Valence, F.; Coton, M.; Thierry, A.; Coton, E. Antifungal Microbial agents for food biopreservation-A review. Microorganisms 2017, 5, 3 .

9. European Food Safety Authority. Introduction of a qualified presumption of safety (QPS) approach for assessment of selected microorganisms referred to EFSA. EFSA J. 2007, 587, 1-16.

10. Varsha, K.K.; Nampoothiri, K.M. Appraisal of lactic acid bacteria as protective cultures. Food Control 2016, 69, 61-64. [CrossRef]

11. Messens, W.; de Vuyst, L. Inhibitory substances produced by Lactobacilli isolated from sourdoughs-A review. Int. J. Food Microbiol. 2002, 72, 31-43. [CrossRef]

12. Crowley, S.; Mahony, J.; Van Sinderen, D. Current perspectives on antifungal lactic acid bacteria as natural bio-preservatives. Trends Food Sci. Technol. 2013, 33, 93-109. [CrossRef] 
13. Le Lay, C.; Coton, E.; Le Blay, G.; Chobert, J.M.; Haertlé, T.; Choiset, Y.; Van Long, N.N.; Meslet-Cladière, L.; Mounier, J. Identification and quantification of antifungal compounds produced by lactic acid bacteria and propionibacteria. Int. J. Food Microbiol. 2016, 239, 79-85. [CrossRef] [PubMed]

14. Cortés-Zavaleta, O.; López-Malo, A.; Hernández-Mendoza, A.; García, H.S. Antifungal activity of lactobacilli and its relationship with 3-phenyllactic acid production. Int. J. Food Microbiol. 2014, 173, 30-35. [CrossRef] [PubMed]

15. Lavermicocca, P.; Valerio, F.; Evidente, A.; Lazzaroni, S.; Corsetti, A.; Gobbetti, M. Purification and characterization of novel antifungal compounds from the sourdough Lactobacillus plantarum strain 21B. Appl. Environ. Microbiol. 2000, 66, 4084-4090. [CrossRef] [PubMed]

16. Hassan, Y.I.; Zhou, T.; Bullerman, L.B. Sourdough lactic acid bacteria as antifungal and mycotoxin-controlling agents. Food Sci. Technol. Int. 2016, 22, 79-90. [CrossRef]

17. Saladino, F.; Luz, C.; Manyes, L.; Fernández-Franzón, M.; Meca, G. In vitro antifungal activity of lactic acid bacteria against mycotoxigenic fungi and their application in loaf bread shelf life improvement. Food Control 2016, 67, 273-277. [CrossRef]

18. Dal Bello, F.; Clarke, C.I.; Ryan, L.A.M.; Ulmer, H.; Schober, T.J.; Ström, K.; Sjögren, J.; van Sinderen, D.; Schnürer, J.; Arendt, E.K. Improvement of the quality and shelf life of wheat bread by fermentation with the antifungal strain Lactobacillus plantarum FST 1.7. J. Cereal Sci. 2007, 45, 309-318. [CrossRef]

19. Rizzello, C.G.; Cassone, A.; Coda, R.; Gobbetti, M. Antifungal activity of sourdough fermented wheat germ used as an ingredient for bread making. Food Chem. 2011, 127, 952-959. [CrossRef] [PubMed]

20. Gerez, C.L.; Torino, M.I.; Rollán, G.; Font de Valdez, G. Prevention of bread mould spoilage by using lactic acid bacteria with antifungal properties. Food Control 2009, 20, 144-148. [CrossRef]

21. Axel, C.; Röcker, B.; Brosnan, B.; Zannini, E.; Furey, A.; Coffey, A.; Arendt, E.K. Application of Lactobacillus amylovorus DSM19280 in gluten-free sourdough bread to improve the microbial shelf life. Food Microbiol. 2015, 47, 36-44. [CrossRef] [PubMed]

22. Axel, C.; Brosnan, B.; Zannini, E.; Peyer, L.C.; Furey, A.; Coffey, A.; Arendt, E.K. Antifungal activities of three different Lactobacillus species and their production of antifungal carboxylic acids in wheat sourdough. Appl. Microbiol. Biotechnol. 2016, 100, 1701-1711. [CrossRef] [PubMed]

23. Le Lay, C.; Mounier, J.; Vasseur, V.; Weill, A.; Le Blay, G.; Barbier, G.; Coton, E. In vitro and in situ screening of lactic acid bacteria and propionibacteria antifungal activities against bakery product spoilage molds. Food Control 2016, 60, 247-255. [CrossRef]

24. Coda, R.; Cassone, A.; Rizzello, C.G.; Nionelli, L.; Cardinali, G.; Gobbetti, M. Antifungal activity of Wickerhamomyces anomalus and Lactobacillus plantarum during sourdough fermentation: Identification of novel compounds and long-term effect during storage of wheat bread. Appl. Environ. Microbiol. 2011, 77, 3484-3492. [CrossRef] [PubMed]

25. Coda, R.; Rizzello, C.G.; Di Cagno, R.; Trani, A.; Cardinali, G.; Gobbetti, M. Antifungal activity of Meyerozyma guilliermondii: Identification of active compounds synthesized during dough fermentation and their effect on long-term storage of wheat bread. Food Microbiol. 2013, 33, 243-251. [CrossRef] [PubMed]

26. Coda, R.; Rizzello, C.G.; Nigro, F.; De Angelis, M.; Arnault, P.; Gobbetti, M. Long-term fungal inhibitory activity of water-soluble extracts of Phaseolus vulgaris cv. Pinto and sourdough lactic acid bacteria during bread storage. Appl. Environ. Microbiol. 2008, 74, 7391-7398. [CrossRef] [PubMed]

27. Rizzello, C.G.; Lavecchia, A.; Gramaglia, V.; Gobbetti, M. Long-term fungal inhibition by Pisum sativum flour hydrolysate during storage of wheat flour bread. Appl. Environ. Microbiol. 2015, 81, 4195-4206. [CrossRef] [PubMed]

28. Russo, P.; Arena, M.P.; Fiocco, D.; Capozzi, V.; Drider, D.; Spano, G. Lactobacillus plantarum with broad antifungal activity: A promising approach to increase safety and shelf-life of cereal-based products. Int. J. Food Microbiol. 2017, 247, 48-54. [CrossRef] [PubMed]

29. Capozzi, V.; Menga, V.; Digesu, A.M.; De Vita, P.; van Sinderen, D.; Cattivelli, L.; Fares, C.; Spano, G. Biotechnological production of vitamin B2-enriched bread and pasta. J. Agric. Food Chem. 2011, 59, 8013-8020. [CrossRef] [PubMed]

30. Axel, C.; Zannini, E.; Arendt, E.K. Mold spoilage of bread and its biopreservation: A review of current strategies for bread shelf life extension. Crit. Rev. Food Sci. Nutr. 2017, 57, 3528-3542. [CrossRef] [PubMed]

31. Gerez, C.L.; Torino, M.I.; Obregozo, M.D.; Font de Valdez, G. A ready-to-use antifungal starter culture improves the shelf life of packaged bread. J. Food Prot. 2010, 73, 758-762. [CrossRef] [PubMed] 
32. Garofalo, C.; Zannini, E.; Aquilanti, L.; Silvestri, G.; Fierro, O.; Picariello, G.; Clementi, F. Selection of sourdough lactobacilli with antifungal activity for use as biopreservatives in bakery products. J. Agric. Food Chem. 2012, 60, 7719-7728. [CrossRef] [PubMed]

33. Axel, C.; Brosnan, B.; Zannini, E.; Furey, A.; Coffey, A.; Arendt, E.K. Antifungal sourdough lactic acid bacteria as biopreservation tool in quinoa and rice bread. Int. J. Food Microbiol. 2016, 239, 86-94. [CrossRef] [PubMed]

34. Russo, P.; Capozzi, V.; Arena, M.P.; Spadaccino, G.; Dueñas, M.T.; López, P.; Fiocco, D.; Spano, G. Riboflavin-overproducing strains of Lactobacillus fermentum for riboflavin-enriched bread. Appl. Microbiol. Biotechnol. 2014, 98, 3691-3700. [CrossRef] [PubMed]

35. Makhoul, S.; Romano, A.; Capozzi, V.; Spano, G.; Aprea, E.; Cappellin, L.; Benozzi, E.; Scampicchio, M.; Märk, T.D.; Gasperi, F.; et al. Volatile compound production during the bread-making process: Effect of flour, yeast and their interaction. Food Bioprocess Technol. 2015, 8, 1925-1937. [CrossRef]

36. Capozzi, V.; Makhoul, S.; Aprea, E.; Romano, A.; Cappellin, L.; Sanchez Jimena, A.; Spano, G.; Gasperi, F.; Scampicchio, M.; Biasioli, F. PTR-MS characterization of VOCs associated with commercial aromatic bakery yeasts of wine and beer origin. Molecules 2016, 21, 483. [CrossRef] [PubMed]

37. Ficco, D.B.M.; Saia, S.; Beleggia, R.; Fragasso, M.; Giovanniello, V.; De Vita, P. Milling overrides cultivar, leavening agent and baking mode on chemical and rheological traits and sensory perception of durum wheat breads. Sci. Rep. 2017, 7, 13632. [CrossRef] [PubMed]

38. Lavermicocca, P.; Valerio, F.; Visconti, A. Antifungal activity of phenyllactic acid against molds isolated from bakery products. Appl. Environ. Microbiol. 2003, 69, 634-640. [CrossRef] [PubMed]

(C) 2017 by the authors. Licensee MDPI, Basel, Switzerland. This article is an open access article distributed under the terms and conditions of the Creative Commons Attribution (CC BY) license (http:/ / creativecommons.org/licenses/by/4.0/). 